Bull. Korean Math. Soc. 51 (2014), No. 2, pp. 511-517

http://dx.doi.org/10.4134/BKMS.2014.51.2.511

\title{
LIGHT 3-CYCLES IN 1-PLANAR GRAPHS WITH DEGREE RESTRICTIONS
}

\author{
XiN ZHANG
}

\begin{abstract}
In this paper, we prove that the 3-cycle is light in the family of 1-planar graphs with minimum vertex degree at least 5 and minimum edge degree at least 12. This generates a known result of Fabrici and Madaras [8].
\end{abstract}

\section{Introduction}

All graphs considered in the paper are finite, simple and undirected. We use $V(G), E(G), F(G), \delta(G)$ and $\Delta(G)$ to denote the set of vertices, the set of edges, the set of faces, the minimum degree and the maximum degree of a plane graph $G$, respectively. The degree of an edge $u v$ in $G$ is the value of $d_{G}(u)+d_{G}(v)$. A $k-, k^{+}$- and $k^{-}$-vertex (resp. face) is a vertex (resp. face) of degree $k$, at least $k$ and at most $k$, respectively. For other undefined concepts we refer the reader to [2].

A graph is 1-planar if it can be drawn on a plane so that each edge is crossed by at most one other edge. The notion of 1-planarity was introduced by Ringel [14], who proved that each 1-planar graph is vertex 7-colorable. This is the first result on the colorings of 1-planar graphs, and from then on, many authors started to investigate the coloring problems (see [1, 3, 4, 6, 15, 16, 17, 21, 23, 24]) and the structural properties (see $[5,8,9,10,11,13,18,19,20,22]$ ) of 1-planar graphs. One of possible approaches in the study of local graph structures can be formalized in the following way (see [12]):

Let $H$ be a connected graph and $\mathcal{G}$ be a family of graphs. If for any graph $G \in \mathcal{G}, G$ contains a subgraph $K \simeq H$ such that $\max _{x \in V(K)}\left\{d_{G}(x)\right\}$ is bounded above by a constant independent of $G$, then we say that $H$ is light in $\mathcal{G}$; otherwise $H$ is heavy in $\mathcal{G}$. By $\mathcal{L}(\mathcal{G})$, we denote the set of light graphs in the family $\mathcal{G}$, and by $\mathcal{P}_{\delta}^{1}(\varepsilon)$, we denote the family of 1 -planar graphs with minimum vertex degree at least $\delta$ and minimum edge degree at least $\varepsilon$.

In 2007, Fabrici and Madaras [8] completely determined the set of light graphs in the family $\mathcal{P}_{4}^{1}(8)$; they are $P_{1}, P_{2}$ and $P_{3}$. Recently, the set of light

Received December 13, 2012; Revised June 12, 2013.

2010 Mathematics Subject Classification. Primary 05C75; Secondary 05C10.

Key words and phrases. 1-planar graph, light graph, triangle, discharging. 
graphs in the family $\mathcal{P}_{5}^{1}(10)$ is also completely determined (see $\left.[8,7,19]\right)$; they are $P_{1}, P_{2}, P_{3}, P_{4}$ and $S_{3}$, but $\mathcal{L}\left(\mathcal{P}_{6}^{1}(12)\right)$ and $\mathcal{L}\left(\mathcal{P}_{7}^{1}(14)\right)$ are still undetermined. In the reference [8], Fabrici and Madaras proved that the 3 -cycle $C_{3}$ is light in $\mathcal{P}_{6}^{1}(12)$. As $\mathcal{P}_{5}^{1}(12)$ is a superfamily of $\mathcal{P}_{6}^{1}(12)$, we are to prove the following main theorem in this paper, which generates the result of Fabrici and Madaras.

Theorem 1. Each 1-planar graph with minimum vertex degree at least 5 and minimum edge degree at least 12 contains a 3-cycle $C=\left[x_{1} x_{2} x_{3}\right]$ such that $\min \left\{d\left(x_{1}\right), d\left(x_{2}\right), d\left(x_{3}\right)\right\} \leq 7$ and $\max \left\{d\left(x_{1}\right), d\left(x_{2}\right), d\left(x_{3}\right)\right\} \leq 20$.

Before giving a proof of the above theorem, we introduce some useful notations. Let $G$ be a 1-planar graph. From now on, we always assume that $G$ has been drawn on a plane so that the 1-planarity of $G$ is satisfied. The associated plane graph of $G$, denoted by $G^{\times}$, is the graph obtained from $G$ by turning all crossings of $G$ into new 4 -vertices, and those new 4 -vertices are called false vertices. The face that is incident with no false vertex in $G^{\times}$is called true face, and otherwise, we call it false face.

\section{The proof of Theorem 1}

Suppose that $G$ is a counterexample to the Theorem 1 and $G^{\times}$is the associated plane graph of $G$. Assign an initial charge $c$ to each element $x \in$ $V\left(G^{\times}\right) \bigcup F\left(G^{\times}\right)$as follows:

$$
c(x)= \begin{cases}d_{G^{\times}}(x)-6, & \text { if } x \in V\left(G^{\times}\right) ; \\ 2 d_{G^{\times}}(x)-6, & \text { if } x \in F\left(G^{\times}\right),\end{cases}
$$

By Euler's formula on $G^{\times}, \sum_{x \in V\left(G^{\times}\right) \cup F\left(G^{\times}\right)} c(x)<0$. We now redistribute the charges among $V\left(G^{\times}\right) \cup F\left(G^{\times}\right)$according to the rules defined below. Before stating them, we introduce some useful notations. In what follows, a special 4 -face is a 4 -face in $G^{\times}$that is incident with two 4 -vertices and two 5 -vertices. By big, mid and small vertices, we denote the vertices $v$ in $G^{\times}$ with $d(v) \geq 21,6 \leq d(v) \leq 20$ and $4 \leq d(v) \leq 5$, respectively. Let $v_{1}, v_{2}$ and $v_{3}$ be three consecutive neighbors of a big vertex $v$ in $G^{\times}$. If $v_{1}$ and $v_{3}$ are both 4 -vertices and $v_{2}$ is a 5 -vertex with $v_{1} v_{2}, v_{2} v_{3} \in E\left(G^{\times}\right)$, then $v_{2}$ is called a special 5-vertex that is adjacent to $v$. The discharging rules are as follows.

Rule 1 Each mid vertex $v$ sends $1-\frac{6}{d_{G} \times(v)}$ to each of its incident faces;

Rule 2 Each big vertex $v$ sends $\frac{2}{3}$ to each of its incident faces;

Rule 3 Each big vertex $v$ sends $\frac{8}{63}$ to each of its adjacent special 5 -vertices;

Rule 4 Each 3 -face $f$ with positive charge $c_{2}(f)$ after applications of Rules 1 and 2 sends $\frac{2}{3} c_{2}(f)$ to each of its incident 4 -vertices, $\frac{1}{3} c_{2}(f)$ to each of its incident 5 -vertices if $f$ is incident with two small vertices, or $c_{2}(f)$ to the unique small vertex that is incident with $f$ if $f$ is incident with exactly one small vertex;

Rule 5 Each special 4 -face sends $\frac{2}{3}$ to each of its incident 4 -vertices and $\frac{1}{3}$ to each of its incident 5 -vertices; 
Rule 6 Each non-special $4^{+}$-face with positive charge after applications of Rules 1 and 2 redistributes this charge uniformly among its incident small vertices.

Let $c^{\prime}(x)$ be the final charge of $x \in V\left(G^{\times}\right) \bigcup F\left(G^{\times}\right)$after applications of the above rules. We now prove that $c^{\prime}(x) \geq 0$ for each $x \in V\left(G^{\times}\right) \cup F\left(G^{\times}\right)$, therefore, $\sum_{x \in V\left(G^{\times}\right) \cup F\left(G^{\times}\right)} c(x)=\sum_{x \in V\left(G^{\times}\right) \cup F\left(G^{\times}\right)} c^{\prime}(x) \geq 0$, which is a contradiction.

Since no two 4 -vertices and no two 5 -vertices are adjacent in $G^{\times}$, any 3 -face $f$ in $G^{\times}$is incident with at most one 4 -vertex and at most one 5 -vertex. Hence, by Rule $4, f$ has a nonnegative final charge. By Rules 1,5 and 6 , it is also easy to check that the final charges of all mid vertices and all $4^{+}$-faces are nonnegative. Thus in the following, we only need estimate the final charges of the small vertices and big vertices.

Claim 1. Each non-special $4^{+}$-face in $G^{\times}$sends at least $\frac{2}{3}$ to each of its incident small vertices.

Proof. Let $f$ be a non-special $4^{+}$-face in $G^{\times}$. Note that after applications of Rules 1 and 2 , the saved charge $c_{2}(f)$ of $f$ is at least $c(f)=2 d(f)-6>0$. If $d_{G \times}(f)=4$, then the number of small vertices that are incident with $f$ is at most 3 , since no two 4 -vertices and no two 5 -vertices are adjacent in $G^{\times}$, thus by Rule $6, f$ sends at least $\frac{2 \times 4-6}{3}=\frac{2}{3}$ to each of its incident small vertices. If $d_{G \times}(f) \geq 5$, then it is easier to check that $f$ sends at least $\frac{2 \times 5-6}{5}=\frac{4}{5}>\frac{2}{3}$ to each of its incident small vertices by Rule 6 .

Claim 2. Each true 3 -face in $G^{\times}$sends at least $\frac{17}{21}$ to each of its incident 5 -vertices.

Proof. Let $f=[u v w]$ be a true 3 -face in $G^{\times}$with $d_{G^{\times}}(v)=5$. Since $G$ is a counterexample to the theorem and the minimum edge degree of $G$ is at least 12, $\max \left\{d_{G^{\times}}(v), d_{G^{\times}}(w)\right\} \geq 21$ and $\min \left\{d_{G^{\times}}(v), d_{G^{\times}}(w)\right\} \geq 7$. By Rules 1 and $2, f$ totally receives at least $1-\frac{6}{7}+\frac{2}{3}=\frac{17}{21}$ from $v$ and $w$. This implies that $c_{2}(f) \geq 2 \times 3-6+\frac{17}{21}=\frac{17}{21}$ and this charge would be transferred to $v$ by Rule 4.

Claim 3. Each big vertex $v$ is adjacent to at most $\left\lfloor\frac{1}{3} d_{G^{\times}}(v)\right\rfloor$ special 5 -vertices in $G^{\times}$.

Proof. Suppose $d_{G \times}(v)=3 r+s$, where $r$ and $s$ are two nonnegative integers with $0 \leq s<3$. In what follows, we assume that $s>0$. (The case when $s=0$ can be dealt with similarly.) Let $v_{1}, v_{2}, \ldots, v_{3 r+s}$ be the neighbors of $v$ in a clockwise order. We now divide those $3 r+s$ vertices into $r+1$ parts $U_{1}, U_{2}, \ldots, U_{r}, U_{r+1}$ so that $U_{i}=\left\{v_{3 i-2}, v_{3 i-1}, v_{3 i}\right\}$ for $1 \leq i \leq r$ and $U_{r+1}=$ $\left\{v_{3 r+1}, \ldots, v_{3 r+s}\right\}$. By the definitions of $U_{i}$ 's, one can check that $U_{i}$ with $1 \leq i \leq r$ contains at most two special 5-vertices. If $U_{i}$ contains exactly two special 5 -vertices, then $v_{3 i-1}$ must be a 4 -vertex, moreover, the two 5 -vertices 
$v_{3 i-2}$ and $v_{3 i}$ satisfy $v_{3 i-2} v_{3 i-1}, v_{3 i-1} v_{3 i} \in E\left(G^{\times}\right)$and $v_{3 i-2} v_{3 i} \in E(G)$. This contradicts the fact that the minimum edge degree of $G$ is at least 12. Hence, each $U_{i}$ with $1 \leq i \leq r$ contains at most one special 5-vertex. Similarly, $U_{r+1}$ also contains at most one special 5-vertex. If $s=1$ and each $U_{i}$ with $1 \leq$ $i \leq r$ contains exactly one special 5 -vertex, then $d_{G \times}\left(v_{3 r-2}\right)=d_{G \times}\left(v_{3 r}\right)=4$, $d_{G \times}\left(v_{3 r-1}\right)=5$ and $v_{3 r-2} v_{3 r-1}, v_{3 r-1} v_{3 r} \in E\left(G^{\times}\right)$. This implies that $v_{3 r+1}$ is not a special 5-vertex, because otherwise we have $v_{3 r} v_{3 r+1} \in E\left(G^{\times}\right)$, which implies $v_{3 r-1} v_{3 r+1} \in E(G)$, a contradiction. Similarly, we can prove that $v_{3 r+1}$ and $v_{3 r+2}$ are not special 5 -vertices when $s=2$ and each $U_{i}$ with $1 \leq i \leq r$ contains exactly one special 5 -vertex. Therefore, $v$ is adjacent to at most $r$ special 5 -vertices in $G^{\times}$.

In the following, we estimate the final charge of the big vertices $v \in V\left(G^{\times}\right)$. Since Rules 2 and 3 are the only rules that are involved with $v$, and Rules 2 and 3 can be applied to $v$ at most $d_{G^{\times}}(v)$ times and $\left\lfloor\frac{1}{3} d_{G^{\times}}(v)\right\rfloor$ times by Claim 3 , respectively, $c^{\prime}(v) \geq d_{G^{\times}}(v)-6-\frac{2}{3} d_{G^{\times}}(v)-\frac{8}{63}\left\lfloor\frac{1}{3} d_{G^{\times}}(v)\right\rfloor \geq \frac{1}{189}\left(55 d_{G^{\times}}(v)-\right.$ 1134) $\geq \frac{1}{9}>0$ for $d_{G \times}(v) \geq 21$.

Until now, the remaining task is to check the nonnegativity of the final charge of small vertices. First, suppose that $v$ is a 5 -vertex. Let $v_{1}, \ldots, v_{5}$ be the neighbors of $v$ in a clockwise sequence and let $f_{i}$ be the face incident with the path $v_{i} v v_{i+1}$ in $G^{\times}$, where the addition on $i$ are taken modulo 5 . By $B(v), T(v)$ and $F(v)$, we denote the number of $4^{+}$-faces, true 3 -faces and false 3 -faces that are incident with $v$, respectively. If $B(v) \geq 3$, then by Rule 5 and Claim 1, $v$ receives at least $3 \times \frac{1}{3}=1$ from its incident $4^{+}$-faces, which implies that $c^{\prime}(v) \geq 5-6+1=0$. If $B(v)=2$ and $f$ is incident with a non-special $4^{+}$-face, then by Rule 5 and Claim $1, c^{\prime}(v) \geq 5-6+\frac{1}{3}+\frac{2}{3}=0$. If $B(v)=2$ and $f$ is incident with two special 4-faces, then those two 4-faces are adjacent in $G^{\times}$, since otherwise we would find two adjacent 4 -vertices in $G^{\times}$, a contradiction. Without loss of generality, suppose that $f_{1}$ and $f_{2}$ are both special 4 -faces. Since $v_{1}, v_{3}$ are false now and $f_{3}, f_{5}$ are 3 -faces, $v_{4}$ and $v_{5}$ are true, which implies that $f_{4}$ is a true 3 -face. Thus by Claim $2, f_{4}$ sends at least $\frac{17}{21}$ to $v$, so $c^{\prime}(v) \geq 5-6+2 \times \frac{1}{3}+\frac{17}{21}>0$ by Rule 5 . If $B(v)=1$ and $v$ is incident with a true 3 -face, then by Rule 5 , Claims 1 and $2, c^{\prime}(v) \geq 5-6+\frac{1}{3}+\frac{17}{21}>0$. If $B(v)=1$ (here we assume that $f_{5}$ is a $4^{+}$-face incident with $v$ ) and all 3 -faces incident with $v$ are false, then either $v_{2}$ and $v_{4}$ are both 4 -vertices, or $v_{1}, v_{3}$ and $v_{5}$ are all 4 -vertices. If the former case occurs, then $f_{5}$ is non-special and $v_{1}, v_{5}$ are not small. This implies that $f_{5}$ sends at least $\frac{2 d_{G} \times\left(f_{5}\right)-6}{d_{G} \times\left(f_{5}\right)-2} \geq 1$ to $v$ by Rule 6 , and thus $c^{\prime}(v) \geq 5-6+1=0$. If the latter case occurs, then $v, v_{2}$ and $v_{4}$ induce a 3-cycle in $G$ with $d_{G \times}(v)=5$, which implies that $v_{2}$ and $v_{4}$ are $7^{+}$-vertices and at least one of them is big. Without loss of generality, assume that $v_{4}$ is a big vertex, then by Rule $3, v_{4}$ sends $\frac{8}{63}$ to $v$, since $v$ is special. On the other hand, one can check that the saved charge of $f_{1}, f_{2}, f_{3}$ and $f_{4}$ after applying Rules 1 and 2 is at least $\frac{1}{7}, \frac{1}{7}, \frac{2}{3}$ and $\frac{2}{3}$, respectively, and $f_{5}$ sends at least $\frac{1}{3}$ to $v$ 
by Rules 5 and 6 . Therefore, $c^{\prime}(v) \geq 5-6+\frac{8}{63}+2 \times \frac{1}{7} \times \frac{1}{3}+2 \times \frac{2}{3} \times \frac{1}{3}+\frac{1}{3}=0$ by Rule 4. If $B(v)=0$ and $T(v) \geq 2$, then by Claim $2, c^{\prime}(v) \geq 5-6+2 \times \frac{17}{21}>0$. If $B(v)=0, T(v)=1$ and $F(v)=4$ (assume that $f_{5}$ is true and $v_{1}$ is big), then by Rules 2 and $4, f_{1}$ sends at least $\frac{1}{3} \times \frac{2}{3}=\frac{2}{9}$ to $v$, thus by Claim 2 , we still have $c^{\prime}(v) \geq 5-6+\frac{2}{9}+\frac{17}{21}>0$.

Now, suppose that $v$ is a 4 -vertex in $G^{\times}$. By Rule 5 and Claim 1, the final charge of $v$ is nonnegative if $v$ is incident with at least three $4^{+}$-faces, so in the following, we assume that $v$ is incident with at least two 3-faces. Let $v_{1}, v_{2}, v_{3}, v_{4}$ be the neighbors of $v$ in a clockwise order. By $f_{i}$, we denote the face that is incident with $v v_{i}$ and $v v_{i+1}$, where the subscripts are taken modulo 4. By $\tau(f \rightarrow v)$, we denote the charge transferred from $f$ to its incident vertex $v$.

Case 1. $v$ is incident with exactly two 3-faces.

First, suppose that $d_{G^{\times}}\left(f_{1}\right)=d_{G^{\times}}\left(f_{3}\right)=3$. Without loss of generality, assume that $v$ is adjacent to at least two 5-vertices (the opposite case when $v$ is adjacent to at most one 5 -vertex can be dealt with similarly). Since the degree of any neighbor of a 5 -vertex in $G$ is at least 7 , we can assume that $d_{G^{\times}}\left(v_{2}\right)=d_{G^{\times}}\left(v_{3}\right)=5$ and $\min \left\{d_{G^{\times}}\left(v_{1}\right), d_{G^{\times}}\left(v_{4}\right)\right\} \geq 7$. Since $d_{G^{\times}}\left(v_{1}\right) \geq 7$, the saved charge of $f_{1}$ after applying Rules 1 and 2 is at least $\frac{1}{7}$, and two thirds of this charge would be transferred to $v$ by Rule 4 , that is to say, $\tau\left(f_{1} \rightarrow\right.$ $v) \geq \frac{1}{7} \times \frac{2}{3}=\frac{2}{21}$. Similarly, we have $\tau\left(f_{2} \rightarrow v\right) \geq \frac{2}{3}, \tau\left(f_{3} \rightarrow v\right) \geq \frac{2}{21}$ and $\tau\left(f_{4} \rightarrow v\right) \geq \frac{8}{7}$. This implies that $c^{\prime}(v) \geq-2+\frac{2}{21}+\frac{2}{3}+\frac{2}{21}+\frac{8}{7}=0$.

Second, suppose that $d_{G^{\times}}\left(f_{1}\right)=d_{G^{\times}}\left(f_{2}\right)=3$. Since $v_{1}, v_{2}$ and $v_{3}$ induce a 3cycle in $G$, we either have $\max _{1 \leq i \leq 3}\left\{d_{G^{\times}}\left(v_{i}\right)\right\} \geq 21$ or have $\min _{1 \leq i \leq 3}\left\{d_{G^{\times}}\left(v_{i}\right)\right\}$ $\geq 8$. If the former case occurs, then we can assume, without loss of generality, that $d_{G^{\times}}\left(v_{1}\right) \geq 21$. By Rules 2, 4, 5, 6 and Claim 1, we have $\tau\left(f_{1} \rightarrow v\right) \geq$ $\frac{2}{3} \times \frac{2}{3}=\frac{4}{9}, \tau\left(f_{3} \rightarrow v\right) \geq \frac{2}{3}$ and $\tau\left(f_{4} \rightarrow v\right) \geq \frac{1}{3} \times\left(2+\frac{2}{3}\right)=\frac{8}{9}$. This implies that $c^{\prime}(v) \geq-2+\frac{4}{9}+\frac{2}{3}+\frac{8}{9}=0$. If the latter case occurs, then by Rules 1 and $4, \tau\left(f_{1} \rightarrow v\right) \geq 2 \times\left(1-\frac{6}{8}\right)=\frac{1}{2}$ and $\tau\left(f_{2} \rightarrow v\right) \geq 2 \times\left(1-\frac{6}{8}\right)=\frac{1}{2}$, and by Rule 5 and Claim $1, \tau\left(f_{3} \rightarrow v\right) \geq \frac{2}{3}$ and $\tau\left(f_{4} \rightarrow v\right) \geq \frac{2}{3}$. This implies that $c^{\prime}(v) \geq-2+2 \times \frac{1}{2}+2 \times \frac{2}{3}>0$.

Case 2. $v$ is incident with exactly three 3-faces.

Suppose that $f_{1}, f_{2}$ and $f_{3}$ are 3 -faces and $f_{4}$ is a $4^{+}$-face. If $v$ is adjacent to no big vertices, then $v$ is adjacent only to $8^{+}$-vertices, since otherwise a light 3 -cycle is found in $G$, a contradiction. By Rules 1 and $4, \tau\left(f_{i} \rightarrow v\right) \geq$ $2 \times\left(1-\frac{6}{8}\right)=\frac{1}{2}$ for $i=1,2,3$. By Rule 5 and Claim $1, \tau\left(f_{4} \rightarrow v\right) \geq \frac{2}{3}$. This implies that $c^{\prime}(v) \geq-2+3 \times \frac{1}{2}+\frac{2}{3}>0$. Therefore, we consider the case when $v$ is adjacent to at least one big vertex. If $v_{2}$ or $v_{3}$, say $v_{2}$, is big, then either $d_{G^{\times}}\left(v_{1}\right)=d_{G^{\times}}\left(v_{3}\right)=d_{G^{\times}}\left(v_{4}\right)=6$ or $\max \left\{d_{G^{\times}}\left(v_{1}\right), d_{G^{\times}}\left(v_{3}\right), d_{G^{\times}}\left(v_{4}\right)\right\} \geq 7$, since $v_{1}, v_{3}$ and $v_{4}$ induce a 3 -path in $G$. If the former case occurs, by Rules 2 and $4, \tau\left(f_{1} \rightarrow v\right) \geq \frac{2}{3}$ and $\tau\left(f_{2} \rightarrow v\right) \geq \frac{2}{3}$, and by Rule 5 and Claim 1 , $\tau\left(f_{4} \rightarrow v\right) \geq \frac{2}{3}$. This implies that $c^{\prime}(v) \geq-2+3 \times \frac{2}{3}=0$. If the latter 
case occurs, then consider the worst case (one can easily check this fact) when $d_{G \times}\left(v_{1}\right)=d_{G \times}\left(v_{4}\right)=5$ and $d_{G \times}\left(v_{3}\right)=7$. By Rules 1, 2, 3, 5 and Claim 1, we can still estimate that $\tau\left(f_{1} \rightarrow v\right) \geq \frac{2}{3} \times \frac{2}{3}=\frac{4}{9}, \tau\left(f_{2} \rightarrow v\right) \geq \frac{2}{3}+\frac{1}{7}=\frac{17}{21}$, $\tau\left(f_{3} \rightarrow v\right) \geq \frac{1}{7} \times \frac{2}{3}=\frac{2}{21}$ and $\tau\left(f_{4} \rightarrow v\right) \geq \frac{2}{3}$. This implies that $c^{\prime}(v) \geq$ $-2+\frac{4}{9}+\frac{17}{21}+\frac{2}{21}+\frac{2}{3}=\frac{1}{63}>0$.

Case 3. $v$ is incident with four 3-faces.

If $v$ is adjacent only to $8^{+}$-vertices, then by Rules 1 and 4 , each face incident with $v$ sends at least $2 \times\left(1-\frac{6}{8}\right)=\frac{1}{2}$ to $v$, which implies that $c^{\prime}(v) \geq-2+$ $4 \times \frac{1}{2}=0$. If $\min _{1 \leq i \leq 4}\left\{d_{G^{\times}}\left(v_{i}\right)\right\} \leq 7$, then there are at least two big vertices among the neighbors of $v$, because otherwise a light 3-cycle would appear in $G$. Without loss of generality, assume that both $v_{1}$ and $v_{3}$ are big vertices and that $d_{G \times}\left(v_{2}\right)=5$ and $d_{G \times}\left(v_{4}\right) \geq 7$ (other cases can be handled similarly and much more easily). In this case, by Rules 1,2 and 4 , one can estimate that $\min _{i=1,2}\left\{\tau\left(f_{i} \rightarrow v\right)\right\} \geq \frac{2}{3} \times \frac{2}{3}=\frac{4}{9}$ and $\min _{i=3,4}\left\{\tau\left(f_{i} \rightarrow v\right)\right\} \geq \frac{2}{3}+\frac{1}{7}=\frac{17}{21}$. This implies that $c^{\prime}(v) \geq-2+2 \times \frac{4}{9}+2 \times \frac{17}{21}>0$.

Since $C_{3}$ is heavy in the family of 1-planar graphs with minimum vertex degree at least 5 , the bound 12 for the minimum edge degree in Theorem 1 cannot be improved to 10 . In view of this, we end this paper by the following open problem.

Problem 1. Determine whether $C_{3} \in \mathcal{L}\left(\mathcal{P}_{5}^{1}(11)\right)$ or not.

Acknowledgements. The author wants to greatly appreciate the help of both the referees and editorial office concerning improvement to this paper. This work is partially supported by the National Natural Science Foundation of China (No. 11301410, 11201440, 11101243), the Natural Science Basic Research Plan in Shaanxi Province of China (No. 2013JQ1002), the Specialized Research Fund for the Doctoral Program of Higher Education (No. 20130203120021), and the Fundamental Research Funds for the Central Universities (No. K5051370003, K5051370021).

\section{References}

[1] M. O. Albertson and B. Mohar, Coloring vertices and faces of locally planar graphs, Graphs Combin. 22 (2006), no. 3, 289-295.

[2] J. A. Bondy and U. S. R. Murty, Graph Theory with Applications, North-Holland, New York, 1976.

[3] O. V. Borodin, Solution of the Ringel problem on vertex-face coloring of planar graphs and coloring of 1-planar graphs, Metody Diskret. Analiz. 41 (1984), 12-26.

[4] - A new proof of the six color theorem, J. Graph Theory 19 (1995), no. 4, 507-521.

[5] O. V. Borodin, I. G. Dmitriev, and A. O. Ivanova, The height of a cycle of length 4 in 1-planar graphs with minimal degree 5 without triangles, J. Appl. Ind. Math. 3 (2009), no. $1,28-31$.

[6] O. V. Borodin, A. V. Kostochka, A. Raspaud, and E. Sopena, Acyclic colouring of 1-planar graphs, Discrete Appl. Math. 114 (2001), no. 1-3, 29-41.

[7] W. Dong, Light paths of 1-planar graphs with bounded minimum degree, manuscript. 
[8] I. Fabrici and T. Madaras, The structure of 1-planar graphs, Discrete Math. 307 (2007), no. 7-8, 854-865.

[9] D. Hudák and T. Madaras, On local structures of 1-planar graphs of minimum degree 5 and girth 4, Discuss. Math. Graph Theory 29 (2009), no. 2, 385-400.

[10] _ On local properties of 1-planar graphs with high minimum degree, Ars Math. Contemp. 4 (2011), no. 2, 245-254.

[11] D. Hudák and P. Šugerek, Light edges in 1-planar graphs with prescribed minimum degree, Discuss. Math. Graph Theory 32 (2012), no. 3, 545-556.

[12] S. Jendrol', T. Madaras, R. Soták, and Z. Tuza, On light cycles in plane triangulations, Discrete Math. 197/198 (1999), 453-467.

[13] V. P. Korzhik and B. Mohar, Minimal obstructions for 1-immersions and hardness of 1-planarity test, Springer Lecture Notes in Computer Science, 5417, 302-312, 2009.

[14] G. Ringel, Ein sechsfarbenproblem auf der Kugel, Abh. Math. Sem. Univ. Hamburg 29 (1965), 107-117.

[15] X. Zhang, J. Hou, and G. Liu, On total colorings of 1-planar graphs, J. Comb. Optim.; Doi:10.1007/s10878-013-9641-9.

[16] X. Zhang and G. Liu, On edge colorings of 1-planar graphs without adjacent triangles, Inform. Process. Lett. 112 (2012), no. 4, 138-142.

[17] On edge colorings of 1-planar graphs without chordal 5-cycles, Ars Combin. 104 (2012), 431-436.

[18] On the lightness of chordal 4-cycle in 1-planar graphs with high minimum degree, Ars Mathematica Contemporanea 7 (2014), no. 2, 281-291.

[19] X. Zhang, G. Liu, and J.-L. Wu, Structural properties of 1-planar graphs and an application to acyclic edge coloring, Scientia Sinica Mathematica 40 (2010), 1025-1032.

[20] Light subgraphs in the family of 1-planar graphs with high minimum degree, Acta Math. Sin. (Engl. Ser.) 28 (2012), no. 6, 1155-1168.

[21] X. Zhang and J.-L. Wu, On edge colorings of 1-planar graphs, Inform. Process. Lett. 111 (2011), no. 3, 124-128.

[22] X. Zhang, J.-L. Wu, and G. Liu, New upper bounds for the heights of some light subgraphs in 1-planar graphs with high minimum degree, Discrete Math. Theor. Comput. Sci. 13 (2011), no. 3, 9-16.

[23] - List edge and list total coloring of 1-planar graphs, Front. Math. China 7 (2012), no. 5, 1005-1018.

[24] X. Zhang, Y. Yu, and G. Liu, On $(p, 1)$-total labelling of 1-planar graphs, Cent. Eur. J. Math. 9 (2011), no. 6, 1424-1434.

Department of Mathematics

Xidian University

XI'AN, 710071, P. R. CHINA

E-mail address: xzhang@xidian.edu.cn 